\title{
Sobrecarga e qualidade de vida do cuidador familiar do idoso da quarta idade
}

\author{
Overload and quality of life of the family caregivers of the fourth age elderly \\ Sobrecarga y calidade de vida de los cuidadores familiares de cuarta edade ancianos
}

Recebido: 30/07/2021 | Revisado: 07/08/2021 | Aceito: 13/08/2021 | Publicado: 16/08/2021

Joana Kátia de Mendonça Flexa Monteiro
ORCID: https://orcid.org/0000-0002-0011-3692
Universidade Federal Fluminense, Brasil
E-mail: joanakatia@ gmail.com
Selma Petra Chaves Sá
ORCID: https://orcid.org/0000-0001-9878-7179
Universidade Federal Fluminense, Brasil
E-mail: selmapetrasa@ gmail.com
Danielle Rachel Coelho Bezerra
ORCID: https://orcid.org/0000-0001-9411-1489
Universidade Federal Fluminense, Brasil
E-mail: danirachel2@ gmail.com

\begin{abstract}
Resumo
Introdução: Com o envelhecimento, o idoso passa a necessitar de auxílio para exercer suas atividades do cotidiano, que, dependendo de cada família, pode ser necessário a contratação de cuidadores profissionais, mas essa responsabilidade frequentemente recai sobre algum familiar. Objetivo: levantar em publicações nacionais e internacionais em artigos publicados no período de 2015 a 2021 que evidenciam Sobrecarga produzida pelo cuidado e a Qualidade de Vida de cuidadores familiares de idosos longevos, bem como, o fato de a Sobrecarga afetar ou não a Qualidade de Vida. Método: Trata-se de uma revisão integrativa de literatura. Para elaboração da pergunta foi utilizada a estratégia PICo, sendo que o processo de busca de artigos e critérios de inclusão seguiram as recomendações PRISMA. A partir da revisão foi desenvolvida uma tecnologia educacional cuidativa em forma de Cartilha. Discussão: Em relação ao perfil dos participantes, a maioria eram mulheres, 91,7\%. Em relação a Qualidade de Vida dos cuidadores, dos 9 estudos que analisaram esse constructo, $55 \%$ dos informantes a consideram insatisfatória. Acerca da Sobrecarga, 10 estudos avaliaram como os cuidadores se sentiam, em 60\% apresentam Sobrecarga moderada. Considerações finais: Exercer o papel de cuidador afeta diretamente a Qualidade de Vida dos responsáveis, gerado principalmente pela Sobrecarga. Cabe a enfermagem capacitar esses cuidadores familiares para que seja possível a redução da Sobrecarga. O produto proposto é uma Cartilha de Orientações de Enfermagem para o Cuidador de Idosos da Quarta Idade, com itens selecionados após a obtenção e análise das discussões encontradas nesta revisão.
\end{abstract}

Palavras-chave: Cuidadores; Idoso; Qualidade de vida.

\begin{abstract}
Introduction: With aging, the elderly need help to perform their daily activities, which, depending on each family, may be necessary to hire professional caregivers, but this responsibility often falls on a family member. Objective: to survey national and international publications in articles published from 2015 to 2020 that show the Burden produced by the care and Quality of Life of family caregivers of long-lived elderly, as well as the fact that the Burden affects or not the Quality of Life Method: This is an integrative literature review. To prepare the question, the PICo strategy was used, and the process of searching for articles and inclusion criteria followed the PRISMA recommendations. From the review, a care educational technology was developed in the form of a booklet. Discussion: Regarding the profile of the participants, most were women, 91.7\%. Regarding the quality of life of caregivers, of the 9 studies that analyzed this construct, 55\% of the informants considered it unsatisfactory. About Burden, 10 studies evaluated how caregivers felt, $60 \%$ had moderate burden. Final considerations: Exercising the role of caregiver directly affects the Quality of Life of those responsible, mainly generated by Overload. It is up to nursing to train these family caregivers so that it is possible to reduce the burden. The proposed product is a Booklet of Nursing Guidelines for Caregivers of Elderly Elderly People, with items selected after obtaining and analyzing the discussions found in this review.
\end{abstract}

Keywords: Caregivers; Old man; Quality of life.

\section{Resumen}

Introducción: Con el envejecimiento, las personas mayores necesitan ayuda para realizar sus actividades diarias, lo que, dependiendo de cada familia, puede ser necesario contratar cuidadores profesionales, pero esta responsabilidad muchas veces recae en un familiar. Objetivo: relevar publicaciones nacionales e internacionales en artículos 
publicados de 2015 a 2020 que muestren la Carga producida por el cuidado y la Calidad de Vida de los cuidadores familiares de ancianos longevos, así como el hecho de que la Carga afecta o no a la Calidad de Vida. Método: esta es una revisión integradora de la literatura. Para la elaboración de la pregunta se utilizó la estrategia PICo y el proceso de búsqueda de artículos y criterios de inclusión siguió las recomendaciones de PRISMA. A partir de la revisión, se desarrolló una tecnología educativa sobre el cuidado en forma de folleto. Discusión: En cuanto al perfil de los participantes, la mayoría eran mujeres, $91,7 \%$. En cuanto a la calidad de vida de los cuidadores, de los 9 estudios que analizaron este constructo, el 55\% de los informantes lo consideró insatisfactorio. Acerca de Burden, 10 estudios evaluaron cómo se sentían los cuidadores, el 60\% tenía una carga moderada. Consideraciones finales: El ejercicio del rol de cuidador incide directamente en la Calidad de Vida de los responsables, generada principalmente por Sobrecarga. Depende de la enfermería formar a estos cuidadores familiares para que sea posible reducir la carga. El producto propuesto es un Folleto de Guías de Enfermería para Cuidadores de Ancianos Ancianos, con ítems seleccionados luego de obtener y analizar las discusiones encontradas en esta revisión.

Palabras clave: Cuidadores; Anciano; Calidad de vida.

\section{Introdução}

O envelhecimento é algo que faz parte da realidade do mundo, o ser humano nunca antes havia conseguido alcançar uma expectativa de vida tão alta como a do presente século.

Pela primeira vez na história, a maioria das pessoas pode esperar viver até os 60 anos ou mais. Combinados com quedas acentuadas nas taxas de fertilidade, esses aumentos na expectativa de vida ocasionam o rápido envelhecimento das populações no mundo (Organização Mundial de Saúde - OMS, 2015).

No Brasil, as taxas de expectativa de vida também alcançaram patamares elevados, segundo dados do Instituto Brasileiro de Geografia e estatística (IBGE, 2020). Uma pessoa nascida no Brasil em 2019 tinha expectativa de viver, em média, até os 76,6 anos, isso representa um aumento de três meses em relação a 2018 (76,3 anos). A expectativa de vida dos homens passou de 72,8 para 73,1 anos e a das mulheres foi de 79,9 para 80,1 anos.

Dentre a população idosa, uma parcela que cresce atualmente são os idosos longevos, que alcançam a idades superiores a oitenta anos. No ano de 2010, essa população equivalia a 1,52\% da pirâmide etária brasileira, as projeções do IBGE para 2025 apontam que o grupo etário acima de 80 anos equivalerá a aproximadamente 2,46\% da população brasileira (IBGE, 2020).

O crescimento da população idosa, por sua vez, ocasiona uma maior demanda de cuidados. O surgimento de diversas condições patológicas faz com que este idoso se torne frágil, uma vez que esse fator está relacionado ao comprometimento da capacidade clínico-funcional. Muitos são os fatores para um envelhecimento com vulnerabilidades, desde questões intrínsecas ao próprio ser, como também questões sociais, culturais, econômicas e ambientais. No contexto brasileiro, quase $40 \%$ dos idosos possuem alguma doença crônica e $29,8 \%$ possuem duas ou mais, como: diabetes, hipertensão ou artrite. Ou seja, ao todo, cerca de $70 \%$ dos idosos possuem alguma doença crônica (Brasil, 2018).

Desse modo, com o envelhecimento, o idoso passa a necessitar de auxílio para exercer suas atividades do cotidiano, que dependendo de cada família, pode ser necessário a contratação de cuidadores profissionais, tal contratação está relacionada as condições econômicas, mas essa responsabilidade frequentemente recai sobre algum familiar.

O cuidador é a pessoa que presta cuidados à outra que esteja necessitando, por estar acamada, com limitações físicas ou mentais, com ou sem remuneração. O cuidador de idoso convive diariamente com o sujeito na faixa etária acima de 60 e que necessita de cuidado parcial ou total como cuidados higiênicos, na alimentação, administrando medicação e estimulando-o com as atividades reabilitadoras, interagindo, assim, com a equipe terapêutica (Brasil, 2008).

O cuidador pode ser classificado como principal ou primário. O cuidador principal é aquele que assume o maior número de tarefas e é o principal responsável pelo idoso, geralmente este tipo de cuidador é um dos familiares do idoso. O cuidador secundário é aquele que contribui com o cuidador principal com algo relacionado ao cuidado (Sampaio et al, 2018). Tanto o cuidador principal quanto o secundário necessitam de ajuda e orientações para conduzir o cuidado com o seu idoso, 
como também realizar o autocuidado.

A busca pela promoção da autonomia e independência do idoso é tarefa árdua e desgastante para os cuidadores, pois estes passam a realizar tarefas que outrora eram de cunho pessoal e desenvolvido de maneira autônoma pelo idoso. Assim, tem uma tarefa difícil, caracterizada por ser repetitiva, interminável e muitas vezes combinada com outras atividades domésticas e profissionais; resultando em exaustão, isolamento social, desrespeito à higiene, tristeza, ocorrência de doenças como depressão e comprometimento das atividades de lazer (Coura et al., 2015).

As consequências dessas condições podem ter um impacto significativo para com o indivíduo e a sua capacidade de realizar tarefas, especificamente, a tomada de decisão pobre, apatia, falta de concentração, desmotivação e a ansiedade podem prejudicar o desempenho no trabalho, possivelmente resultando em ameaças letais para a segurança do idoso.

Desse modo, a função de cuidador pode gerar uma Sobrecarga, afetando a Qualidade de Vida da pessoa que é responsável pelo cuidado, pois demanda uma dedicação total. Ressalta-se que o cuidado prestado ao idoso pelo cuidador exige dedicação exclusiva e quase sempre integral, que, muitas vezes, leva o cuidador à instalação de uma nova dinâmica de vida, baseada nas necessidades do ser cuidado por ele.

A enfermagem deve trabalhar em equipe, de forma a englobar o cuidador na promoção do cuidado. $O$ enfermeiro deve atuar junto com o cuidador e com a família, auxiliando-os na reorganização das atividades diárias, percebendo as dificuldades, prevenindo e controlando adversidades. É essencial que o cuidador seja reconhecido pela equipe de saúde como parte integral e importante no cuidado, reabilitação e Qualidade de Vida do cliente. Devemos nos atentar que o cuidador possui, além de suas qualidades, limitações e inseguranças e que deve receber assistência das equipes de saúde (Gomes \& Resck, 2009).

Assim, optou-se por buscar na literatura informações acerca da Qualidade de Vida e Sobrecarga do cuidador familiar. Tema esse muito importante, principalmente com o aumento do envelhecimento da população, que precisará de um maior cuidado dos profissionais de enfermagem, tanto na prestação de cuidados diretos para com os idosos, como na orientação dos cuidadores que os acompanham diariamente.

Diante dessa realidade, torna-se essencial o desenvolvimento de estudos e pesquisas centradas na área da enfermagem, com enfoque no cuidador de idoso, especialmente o cuidador familiar, considerando-se que este, no desenvolver do seu papel, expressa sua importância manifestando-se como elo entre a família/idoso e o serviço de saúde. Observa-se que há carências de recursos de suporte formal e implementações de políticas públicas nacionais que amparem esse cuidador, pois na sua rotina diária pode desencadear agravos de saúde.

Prestar cuidado à saúde é uma atividade que exige conhecimento, requer competências e habilidades e, nesse contexto, o cuidador precisa se adaptar e conviver com as mudanças ocorridas na vida do idoso da quarta idade. Dessa maneira, este estudo buscou também descrever o perfil dos responsáveis pelo cuidado com o idoso, bem como, as propostas dos autores para a melhoria da Qualidade de Vida do cuidador.

Logo, o objetivo geral desta pesquisa é: Levantar em publicações nacionais e internacionais evidências acerca da Sobrecarga produzida pelo cuidado e a Qualidade de Vida de cuidadores familiares de idosos longevos. Como objetivos específicos: Discutir os dados encontrados visando buscar implicações para a área da Enfermagem e propor um produto que vise orientar os Cuidadores Familiares de Idosos da Quarta Idade com relação a prevenção da Sobrecarga e estresse.

\section{Metodologia}

Trata-se de uma revisão de literatura com uso do método de revisão integrativa de literatura, que tem como propósito agrupar e sumarizar o conhecimento cientifico sobre o tema pesquisado. Segundo Souza, Silva \& Carvalho (2010), a revisão integrativa é a mais ampla abordagem metodológica referente às revisões e permite a inclusão de estudos experimentais e nãoexperimentais para uma compreensão completa do fenômeno analisado. 
Além disso, o termo revisão integrativa tem origem na integração de opiniões, conceitos ou ideias provenientes das pesquisas utilizadas no método, ponto esse que evidencia o potencial para se construir a ciência. Permitindo a síntese e análise do conhecimento científico já produzido sobre o tema investigado (Grupo Anima Educação, 2014).

A revisão integrativa ao se fundamentar no rigor científico exigido para outras abordagens da pesquisa, representa mais um recurso para a construção do conhecimento em enfermagem e, dado sua natureza, pode subsidiar o desenvolvimento e a acurácia da prática clínica e consequentes intervenções no paciente (Crossetti, 2012). As etapas percorridas pela presente pesquisa foram as recomendadas por Sousa et al. (2017), sendo as seguintes: Na primeira etapa ocorreu a identificação do tema ou questão de pesquisa: o tema escolhido está relacionado a Qualidade de Vida e a Sobrecarga do cuidador familiar do idoso, para a formulação da pergunta e por se tratar de uma pesquisa não clínica, utilizou-se a estratégia PICo (Tabela 1), emergindo a seguinte questão de pesquisa: "quais as informações disponíveis na literatura sobre como a Sobrecarga produzida pelo cuidado ao idoso longevo afeta a Qualidade de Vida do cuidador familiar ?".

Tabela 1. Estratégia PICo para a criação da pergunta de pesquisa.

\begin{tabular}{cc}
\hline P- População & Cuidador do idoso \\
\hline I- Interesse & Qualidade de vida e Sobrecarga \\
\hline Co- Contexto & Familiar \\
\hline
\end{tabular}

Fonte: Autores (2021).

$\mathrm{Na}$ segunda etapa, foram estabelecidos os critérios para inclusão e exclusão dos estudos, os critérios de inclusão foram artigos publicados entre 2015 e 2021, cujo foco foi a Sobrecarga do cuidado ao idoso longevo e as implicações na Qualidade de Vida do cuidador familiar, disponíveis na íntegra no idioma português e inglês, nas bases de dados Literatura LatinoAmericana e do Caribe em Ciências da Saúde (LILACS), por ser uma base de dados especializada na área da saúde, englobando 26 países da América Latina e do Caribe, além de possuir acesso livre gratuito; Medical Literature Analysis and Retrieval Sytem Online (MEDLINE), que é o mais importante banco de dados destinados à pesquisas científica da área biomédica, disponibilizado gratuitamente pela internet; Base de Dados de Enfermagem (BDENF) que é uma fonte de informação compostas por referências bibliográficas da literatura técnico científica brasileira em enfermagem contendo artigos científicos das revistas mais conceituadas da área de Enfermagem; The Scientific Electronic Library Online (SCIELO), além de ter acesso ao texto completo das publicações, é considerado a principal biblioteca digital da América Latina. Como critérios de exclusão: estudos com resultados inconclusivos, pesquisa secundárias, teses, dissertações, artigos duplicados e que possuam como participantes da pesquisa cuidador formal.

As buscas dos estudos ocorreram nos seguintes locais de pesquisa: SCIELO e Biblioteca Virtual De Saúde (BVS), no mês de março de 2021, para o refinamento foram pesquisados Descritores em Ciências da Saúde (DECs) sendo selecionados os seguintes: "Cuidadores", "Idoso", "Qualidade de Vida", e os termos correspondentes em inglês "Caregivers ", "Ageded", "Quality of Life" junto com o operador booleano AND (Tabela 2).

Tabela 2. Distribuição dos artigos encontrados e os selecionados segundo fonte de informação. Niterói, Rio de Janeiro, 2021.

\begin{tabular}{c|cc} 
Fonte da informação & Artigos encontrados & Artigos selecionados \\
\hline MEDLINE & 1.456 & 7 \\
LILACS & 71 & 3 \\
BDENF & 31 & 1 \\
SCIELO & 4 & 1 \\
Total & 1.562 & 12 \\
\hline
\end{tabular}

Fonte: Autores (2021). 
A amostra inicial com o uso dos descritores e qualificador na busca avançada foi de 1.562 estudos, que após a utilização dos filtros presentes nas bases (texto completo, idioma, ano e tipo de arquivo), resultaram em 614 artigos para análise do título, restando 60 artigos para a leitura na integra, sendo incluídos na revisão 12 artigos (Figura 1).

Figura 1. Fluxograma do processo de busca de artigos e critérios de inclusão elaborado segundo recomendações PRISMA.
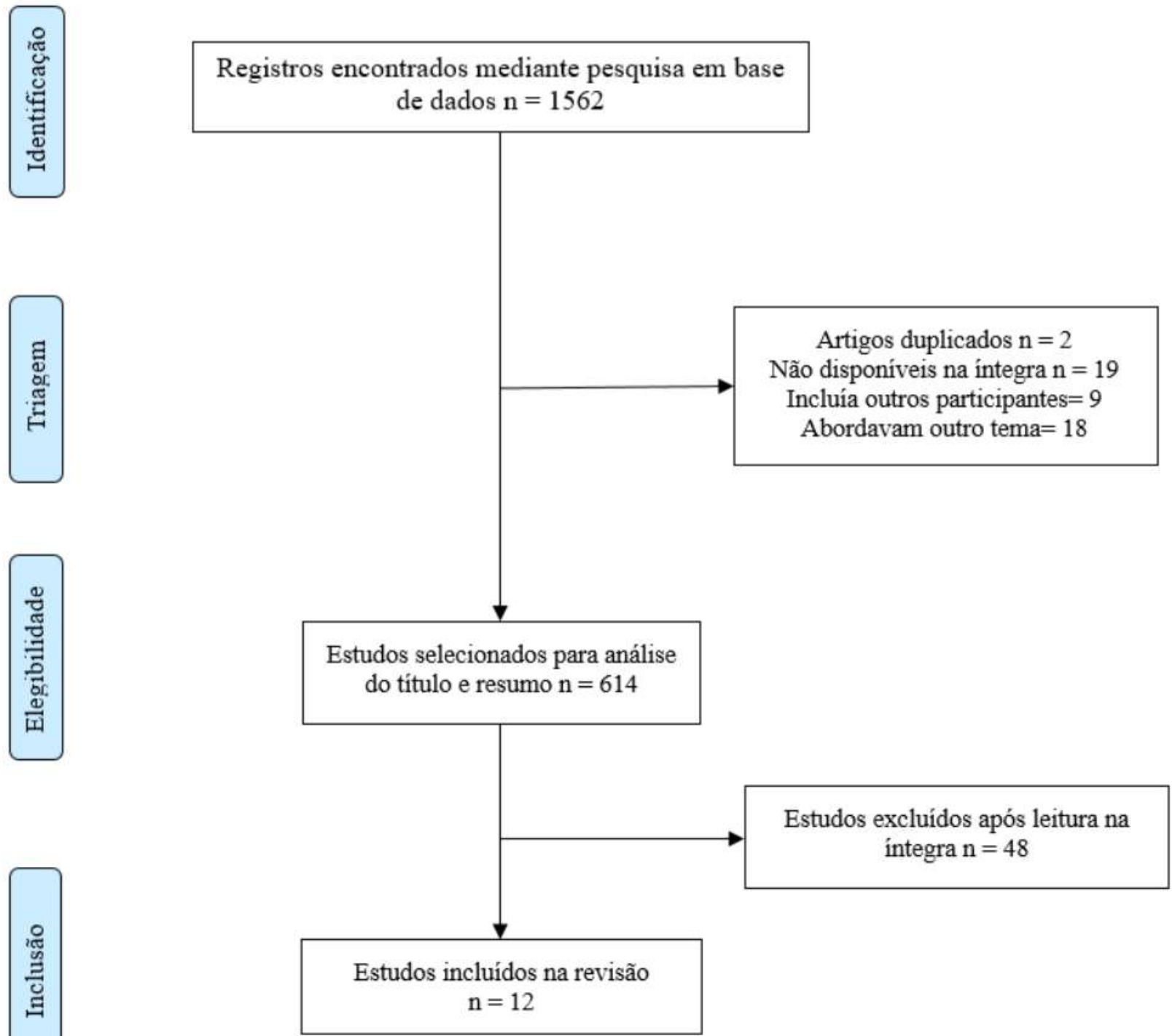

Fonte: Autores (2021).

$\mathrm{Na}$ busca executada, os estudos foram selecionados de acordo com os filtros utilizados nas bases para avaliar se assemelhavam a pergunta norteadora e aos objetivos da pesquisa, além de ter sido analisado os títulos dos artigos, a leitura do resumo, e por último a leitura na íntegra dos estudos.

$\mathrm{Na}$ terceira etapa buscou-se definir as informações a serem extraída dos estudos dos 12 inclusos, visando responder à questão norteadora. Foram criados 2 instrumentos para a melhor análise dos dados extraídos, o primeiro com a identificação e as referências. E o segundo instrumento com autores, título, base de dados e metodologia, ficando no tópico subsequente.

A quarta etapa consistiu na avaliação dos principais resultados e principais conclusões encontradas nos artigos selecionados.

$\mathrm{Na}$ quinta fase, tendo em vista a análise crítica dos estudos elegíveis, adotou-se a classificação da Agency for 
Healthcare Research and Quality (AHRQ) dos Estados Unidos da América, elaborado por estudiosos da enfermagem. A qualidade das evidências é classificada em seis níveis, a saber: nível 1, metanálise de múltiplos estudos controlados; nível 2, estudo individual com delineamento experimental; nível 3, estudo com delineamento quase-experimental como estudo sem randomização com grupo único pré e pós-teste, séries temporais ou caso-controle; nível 4, estudo com delineamento nãoexperimental como pesquisa descritiva correlacional e qualitativa ou estudos de caso; nível 5, relatório de casos ou dado obtido de forma sistemática, de qualidade verificável ou dados de avaliação de programas; nível 6, opinião de autoridades respeitáveis baseada na competência clínica ou opinião de comitês de especialistas, incluindo interpretações de informações não baseadas em pesquisas (Mercês et al, 2019).

A partir da revisão, foi desenvolvida uma Cartilha que é tecnologia educacional cuidativa, que segundo Salbego et al (2018) é um conjunto de saberes/conhecimentos científicos, que sustentam a operacionalização do processo de cuidar e educar do outro de modo direto e indireto na práxis do enfermeiro, a partir da experiência cotidiana e da pesquisa dentro de uma perspectiva crítica, reflexiva, criativa, transformadora e multidimensional, entre os seres humanos e o meio em que se inserem.

A Cartilha foi construída conforme as recomendações para concepção e eficácia para materiais educativos, de acordo com as características: conteúdo, linguagem, organização, layout e ilustração (Hoffmanm \& Warrall, 2004).

Utilizou-se para o planejamento e elaboração da cartilha o conteúdo teórico disponível neste estudo. Usou-se o Manual do Cuidador da Pessoa Idosa da Secretaria Especial dos Direitos Humanos, publicada no ano de 2008, como aporte para esta etapa.

Empregou-se o programa Power Point (versão 2013) para composição do layout e diagramações e as imagens, de domínio público, foram retiradas do Pinterest (br.pinterest.com).

Por fim, contemplou-se ao conteúdo da Cartilha (elementos textuais) os seguintes tópicos: Apresentação: aborda o objetivo do material; O cuidador Familiar de Idosos: traz o conceito sobre este termo; De que maneira o cuidar o idoso da quarta idade afeta a saúde e o bem-estar do cuidador?: este tópico mostra como a falta de conhecimento por parte do cuidador acarreta a Sobrecarga; Cuidador, vamos exercitar o autocuidado?: estímulo para o cuidador cuidar de si próprio; Dicas para prevenir a Sobrecarga do Cuidador: como o próprio título diz, tem o intuito de evitar a Sobrecarga do cuidador nos aspectos físicos, psicológicos/emocionais e sociais; Declaração dos Direitos do Cuidador: tem o objetivo de esclarecer o cuidador a respeito de seus direitos como pessoa; Cuidador fique sabendo: apanhado de itens que serve como reforço para que o cuidador também foque no seu bem estar e não apenas do idoso; Telefones úteis e endereços eletrônicos: para consulta do cuidador em caso de necessidade ou dúvida em relação ao seu autocuidado e cuidado do idoso e Testando a Sobrecarga/estresse do cuidador familiar de idoso: baseado na adaptação da escala Zarit com finalidade de incentivar o cuidador a avaliar seu nível de Sobrecarga e estresse e procurar ajuda profissional, caso seja necessário. 
Figura 2. Representação ilustrativa da cartilha educativa. Niterói, Rio de Janeiro, 2021.

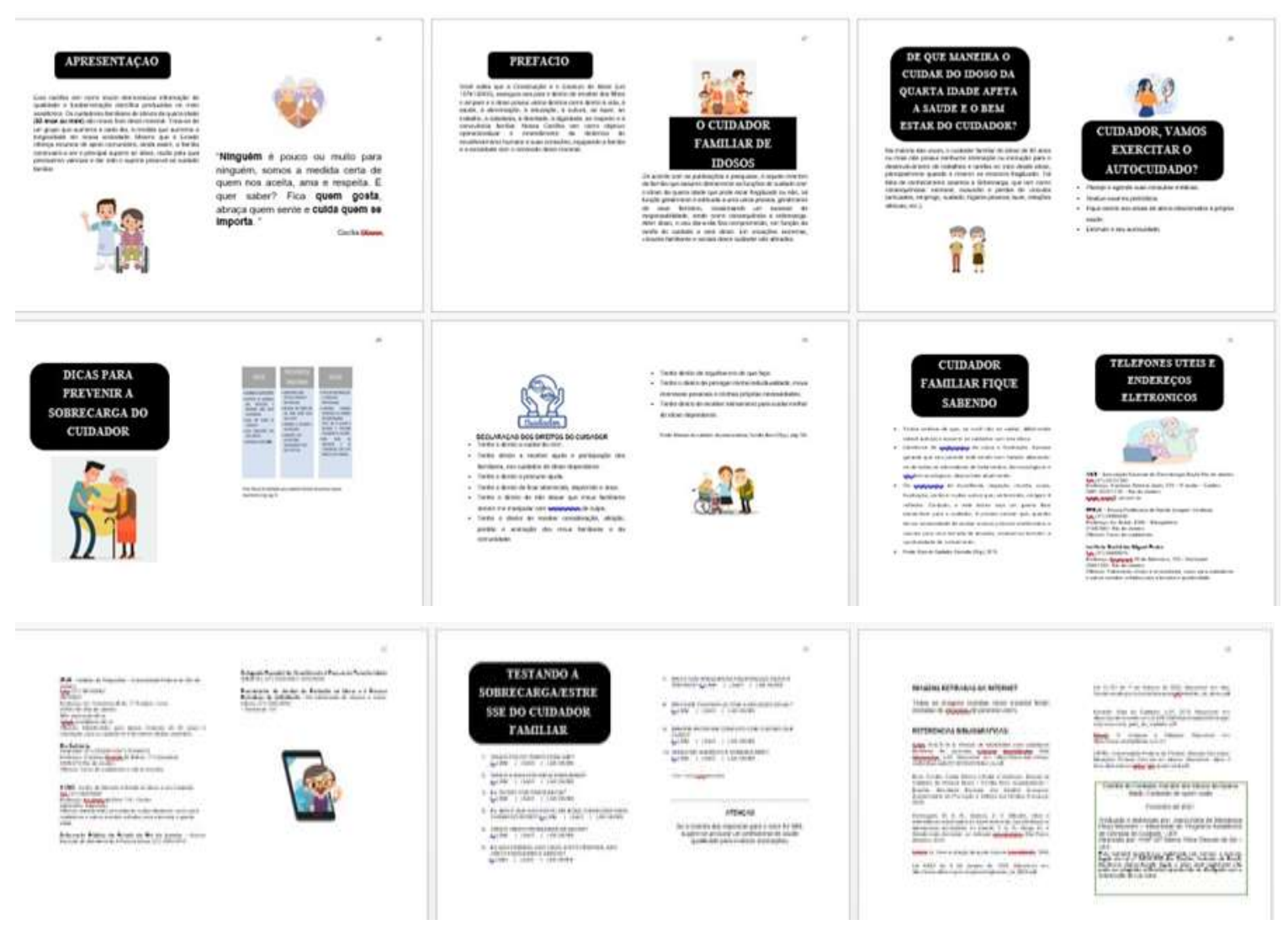

Fonte: Autores (2021).

\section{Resultados}

\section{Caracterização da Amostra da RI}

Dos estudos encontrados relacionados aos descritores pesquisados nas bases de dados, somente $12(0,77 \%)$ corresponderam aos critérios de inclusão propostos. Destes, 4 (33,3\%) publicados no ano de 2019, 3 (25\%) no ano de 2018, e 2 (16,7\%) em 2015, nos anos de 2016, 2017 e 2020 foram encontrados somente uma publicação para cada ano.

Quanto ao local de realização dos estudos, o Brasil se destacou com o maior número de estudos realizados em seu território com 7 (58,4\%), seguido do Japão com 2 estudos (16,7\%), Itália, China e Estados Unidos da América com uma publicação apenas (Tabela 3). O método mais utilizado foi estudo transversal em 10 estudos (83,3\%).

Em relação ao perfil dos participantes, em 11 estudos a maioria eram mulheres (91,7\%), em somente um estudo havia mais homens $(8,3 \%)$, quanto a idade dos cuidadores, a faixa etária mais encontrada foi entre 50 a 59 anos em 5 estudos (41,7\%), seguida de pessoas entre 60 a 69 anos em 3 estudos (25\%), em 2 (16,7\%) estudos pessoas com 40 a 49 anos e um não descreveu essa informação.

Já sobre a escolaridade destes cuidadores, grande parte possui o ensino fundamental completo ou incompleto descrito em 5 estudos (41,7\%), e estado civil casado em $11(91,7 \%)$.

Da relação com o idoso cuidado, 6 estudos (50\%) eram parentes de primeiro grau (filho (a), pai ou mãe), em 3 (25\%) estudos eram cônjuges, e 4 (33,3\%) não descreveram a relação. Acerca da idade do idoso cuidado, a faixa etária mais frequente foi de idosos octogenários, citados em 5 (41,7\%), e a demência foi uma doença muito mencionada entre esses idosos, citados em $5(41,7 \%)$. 
Tabela 3. Artigos que compuseram a amostra da RI de acordo com o título, ano, periódico e país. Niterói. Rio de Janeiro, 2021.

\begin{tabular}{|c|c|c|c|c|}
\hline $\mathbf{N}^{\mathbf{o}}$ & Título & Ano & Fonte & $\begin{array}{c}\text { País de realização do } \\
\text { estudo }\end{array}$ \\
\hline 1 & $\begin{array}{c}\text { Elderly Who Care for Elderly:Double Vulnerability and } \\
\text { Quality of Life }\end{array}$ & 2020 & Paidéia & Brasil \\
\hline 2 & $\begin{array}{l}\text { Cuidando de quem cuida: Qualidade de Vida e Sobrecarga } \\
\text { de mulheres cuidadoras }\end{array}$ & 2019 & $\begin{array}{l}\text { Rev. Bras. } \\
\text { Enferm. }\end{array}$ & Brasil \\
\hline 3 & $\begin{array}{l}\text { Cuidado domiciliar a idosos dependentes de cuidadores } \\
\text { familiares com Sobrecarga e desconforto emocional }\end{array}$ & 2019 & $\begin{array}{l}\text { J. res.: } \\
\text { fundam. care. }\end{array}$ & Brasil \\
\hline 4 & $\begin{array}{c}\text { Family Caregivers' Perspectives for the Effect of Social } \\
\text { Support on their Care Burden and Quality of Life: A } \\
\text { Mixed-Method Study in Rural and Sub-Urban Central } \\
\text { Japan }\end{array}$ & 2019 & $\begin{array}{l}\text { Tohoku J Exp } \\
\text { Med }\end{array}$ & Japão \\
\hline 5 & $\begin{array}{c}\text { Quality of Life of Caregivers of Older Patients with } \\
\text { Advanced Cancer }\end{array}$ & 2019 & $\begin{array}{l}\text { J Am Geriatr } \\
\text { Soc. }\end{array}$ & Estados Unidos \\
\hline 6 & Sobrecarga no cuidado de paciente idoso com demência & 2018 & $\begin{array}{l}\text { Revista } \\
\text { Kairós - } \\
\text { Gerontologia }\end{array}$ & Brasil \\
\hline 7 & $\begin{array}{l}\text { Caregiver burden and its related factors in advanced } \\
\text { Parkinson's disease: data from the PREDICT study. }\end{array}$ & 2018 & $\begin{array}{l}\text { Journal of } \\
\text { Neurology }\end{array}$ & Itália \\
\hline 8 & $\begin{array}{l}\text { Alzheimer's disease severity and its association with } \\
\text { patient and caregiver quality of life in Japan: results of a } \\
\text { community-based survey. }\end{array}$ & 2018 & BMC Geriatr & Japão \\
\hline 9 & $\begin{array}{l}\text { Factors associated with health-related quality of life among } \\
\text { Family caregivers of disabled older adults: a cross-sectional } \\
\text { study from Beijing. }\end{array}$ & 2017 & Medicine & China \\
\hline 10 & $\begin{array}{l}\text { Prevalência de Sobrecarga e respectivos fatores associados } \\
\text { em cuidadores de idosos dependentes, em uma região pobre } \\
\text { do Rio de Janeiro, Brasil }\end{array}$ & 2016 & $\begin{array}{l}\text { Cadernos de } \\
\text { Saúde Pública }\end{array}$ & Brasil \\
\hline 11 & $\begin{array}{l}\text { Associação entre apoio social e Qualidade de Vida } \\
\text { de cuidadores familiares de idosos dependentes. }\end{array}$ & 2015 & $\begin{array}{l}\text { Ciência \& } \\
\text { Saúde } \\
\text { Coletiva } \\
\end{array}$ & Brasil \\
\hline 12 & $\begin{array}{l}\text { Quality of life of caregivers of } \\
\text { octogenarians: a study using the } \\
\text { WHOQOL-BREF }\end{array}$ & 2015 & $\begin{array}{l}\text { Invest Educ } \\
\text { Enferm }\end{array}$ & Brasil \\
\hline
\end{tabular}

Fonte: Autores (2021).

Quanto a análise da Sobrecarga e Qualidade de Vida, 3 (25\%) estudos apresentaram apenas a Sobrecarga (3,5, 10), 2 $(16,7 \%$ \%) pesquisaram apenas a Qualidade de Vida $(11,12)$, os demais analisaram ambas as informações. Em relação a Qualidade de Vida dos cuidadores, dos 9 estudos que analisaram esse constructo, em 5 (55\%) os informantes a consideram insatisfatória $(1,4,9,11,12)$, em $3(33 \%)$ mediana $(2,6,8)$ e 1 (11\%) consideraram a Qualidade de Vida boa (7).

Acerca da Sobrecarga, 10 estudos avaliaram como os cuidadores se sentiam, em 6 estudos (60\%) sobrecarga moderada $(2,3,4,8,9,10), 2(20 \%)$ sobrecarga alta $(1,5)$ e $2(20 \%)$ sobrecarga baixa $(6,7)$. Além disso, dentre as dificuldades relatadas pelos cuidadores que afetavam a vida, $7(58,4 \%)$ relataram falta de apoio e tempo para si $(3,4,6,7,9,10,12), 6$ $(50 \%)$ apresentavam alguma comorbidade $(1,3,4,5,8,9), 4(33,3 \%)$ sobrecarga financeira $(4,7,11,12)$. No quadro abaixo estão as principais informações extraídas dos artigos, sendo enumeradas de 1 a 12 (Quadro 1). 
Quadro 1. Informações acerca dos estudos publicados sobre a Qualidade de Vida e Sobrecarga entre os anos de 2015 a 2020 de acordo com autores, objetivo, método, principais resultados, principais conclusões e nível de evidência. Niterói. Rio de Janeiro, 20201

\begin{tabular}{|c|c|c|c|c|c|}
\hline $\mathbf{N}^{\mathbf{o}}$ & AUTORES & OBJETIVO/MÉTODO & PRINCIPAIS RESULTADOS & $\begin{array}{l}\text { PRICINCIPAIS } \\
\text { CONCLUSÕES }\end{array}$ & $\begin{array}{c}\text { NÍVEL DE } \\
\text { EVIDÊNCIA }\end{array}$ \\
\hline 1 & Flesch et al. & $\begin{array}{c}\text { Identificar a associação entre a } \\
\text { dupla vulnerabilidade e a } \\
\text { Qualidade de Vida de cuidadores } \\
\text { de idosos. } \\
\text { Método: qualitativo. }\end{array}$ & $\begin{array}{l}\text { A análise multivariada hierarquizada } \\
\text { mostrou que os cuidadores de idosos com } \\
\text { maior risco de pior Qualidade de Vida eram } \\
\text { aqueles com três ou mais doenças, com } \\
\text { Sobrecarga média ou alta e com pior } \\
\text { avaliação de saúde em relação ao } \\
\text { passado. Espera-se maior debilidade na } \\
\text { saúde ao longo do envelhecimento. }\end{array}$ & $\begin{array}{l}\text { O número de cuidadores } \\
\text { idosos vem aumentando e } \\
\text { apresentam especificidades } \\
\text { diferentes dos cuidadores de } \\
\text { outras faixas etárias. }\end{array}$ & IV \\
\hline 2 & Araújo et al. & $\begin{array}{l}\text { Avaliar a Qualidade de Vida e } \\
\text { Sobrecarga de mulheres } \\
\text { cuidadoras. } \\
\text { Método quantitativo, descritivo, } \\
\text { transversal. }\end{array}$ & $\begin{array}{c}\text { Observa-se que não houve diferença } \\
\text { estatística significante entre os domínios de } \\
\text { QV e os níveis de Sobrecarga. Entretanto, } \\
\text { identificou-se correlação negativa de } \\
\text { intensidade moderada (-0,383) entre o } \\
\text { somatório das questões de QV e o de } \\
\text { Sobrecarga. }\end{array}$ & $\begin{array}{l}\text { Quanto maior a pontuação } \\
\text { atingida na QV, menor será a } \\
\text { Sobrecarga ou vice-versa. }\end{array}$ & V \\
\hline 3 & $\begin{array}{l}\text { Couto; Caldas } \\
\text { \& Castro }\end{array}$ & $\begin{array}{l}\text { Compreender as experiências de } \\
\text { cuidadores familiares com } \\
\text { Sobrecarga e desconforto } \\
\text { emocional ao cuidarem de idosos } \\
\text { dependentes no domicílio. } \\
\text { Método: qualitativo. }\end{array}$ & $\begin{array}{l}\text { Substantivamente obteve-se que o idoso } \\
\text { dependente com necessidades de cuidados } \\
\text { no domicílio estimulou movimento na } \\
\text { família, pelo qual um familiar tornou-se } \\
\text { cuidador. Com a vivência desse papel, } \\
\text { desenvolveu Sobrecarga, desgaste } \\
\text { emocional, repercutindo na Qualidade de } \\
\text { Vida, demandando atenção, apoio e } \\
\text { capacitação. }\end{array}$ & $\begin{array}{c}\text { Cuidadores familiares } \\
\text { necessitam de recursos } \\
\text { assistenciais do sistema de } \\
\text { saúde, suporte emocional e } \\
\text { aprendizagem de processos. }\end{array}$ & $\mathrm{V}$ \\
\hline 4 & Haya et al & $\begin{array}{c}\text { Avaliação dos cuidadores } \\
\text { familiares sobre o apoio social, o } \\
\text { tipo e a fonte de apoio } \\
\text { considerados importantes e como } \\
\text { isso afetou a Sobrecarga do } \\
\text { cuidado e a Qualidade de Vida. } \\
\text { (QV). } \\
\text { Método: qualitativo- } \\
\text { quantitativo. }\end{array}$ & $\begin{array}{l}\text { Forte apoio familiar e educação superior } \\
\text { tiveram efeitos positivos na QV, enquanto } \\
\text { maior carga de cuidado e maior duração do } \\
\text { cuidado tiveram efeitos negativos na QV. }\end{array}$ & $\begin{array}{l}\text { Os cuidadores perceberam o } \\
\text { suporte de forma positiva, } \\
\text { mas os efeitos dependeram de } \\
\text { quem forneceu o suporte. } \\
\text { Uma vez que, quando o apoio } \\
\text { vem de familiares próximos e } \\
\text { profissionais, é percebido de } \\
\text { maneira positiva, já quando o } \\
\text { apoio vem de vizinhos ou } \\
\text { parentes distantes, devemos } \\
\text { considerar as necessidades e } \\
\text { condições dos cuidadores, } \\
\text { afim de evitar um impacto } \\
\text { negativo. }\end{array}$ & IV \\
\hline 5 & Kehoe et al. & $\begin{array}{l}\text { Avaliar as relações entre os } \\
\text { domínios relacionados ao } \\
\text { envelhecimento capturados pela } \\
\text { avaliação geriátrica (AG) para } \\
\text { pacientes mais velhos com câncer } \\
\text { avançado e a saúde emocional e a } \\
\text { Qualidade de Vida (QV) dos } \\
\text { cuidadores. } \\
\text { Método: qualitativo. }\end{array}$ & $\begin{array}{l}\text { Menor idade do cuidador, comorbidade do } \\
\text { cuidador e sofrimento do paciente foram } \\
\text { associados a piores resultados do cuidador. }\end{array}$ & $\begin{array}{l}\text { As deficiências dos pacientes } \\
\text { geriátricos foram associados } \\
\text { a pior saúde emocional e } \\
\text { menor Qualidade de Vida dos } \\
\text { cuidadores. }\end{array}$ & IV \\
\hline 6 & Dias et al. & $\begin{array}{l}\text { Avaliar o nível de Sobrecarga dos } \\
\text { cuidadores dos pacientes com } \\
\text { demência e seus fatores, } \\
\text { caracterizar a amostra de } \\
\text { pacientes e cuidadores. } \\
\text { Método: transversal, } \\
\text { qualitativo-quantitativo }\end{array}$ & $\begin{array}{l}\text { Dentre os cuidadores avaliados, prevaleceu } \\
\text { o gênero feminino, com média de idade de } \\
53 \pm 12,4 \text { e redução da realização de } \\
\text { atividades de lazer, pelos cuidadores, antes e } \\
\text { após de assumirem o papel de cuidador } \\
\text { informal. A análise de correlação de } \\
\text { Spearman mostrou correlação entre o } \\
\text { aumento de Sobrecarga e redução da prática } \\
\text { de atividade de lazer ( } \mathrm{p}=0,0078) \text {. }\end{array}$ & $\begin{array}{l}\text { A redução ou anulação da } \\
\text { atividade de lazer na vida } \\
\text { cotidiana implica na } \\
\text { Qualidade de Vida desses } \\
\text { cuidadores, o que pode ser } \\
\text { evidenciado pela Sobrecarga } \\
\text { apresentada. }\end{array}$ & IV \\
\hline
\end{tabular}




\begin{tabular}{|c|c|c|c|c|c|}
\hline 8 & $\begin{array}{l}\text { Montgomery et } \\
\text { al. }\end{array}$ & $\begin{array}{c}\text { Investigar as associações entre a } \\
\text { gravidade da doença do paciente } \\
\text { com demência de Alzheimer a } \\
\text { Qualidade de Vida relacionada à } \\
\text { saúde (QVRS) de ambos os } \\
\text { pacientes (relatório proxy) e seus } \\
\text { cuidadores que vivem no Japão, } \\
\text { bem como, comorbidades } \\
\text { relacionadas ao cuidado, como } \\
\text { depressão. } \\
\text { Método: transversal, } \\
\text { quantitativo. }\end{array}$ & $\begin{array}{c}\text { Em } 300 \text { cuidadores entrevistados, os } \\
\text { resultados multivariáveis revelaram que o } \\
\text { aumento da gravidade da demência } \\
\text { (Alzheimer) foi significativamente } \\
\text { associado a piores pontuações do paciente e } \\
\text { do cuidador no EQ-5D e uma alta proporção } \\
\text { de cuidadores (30,0\%) relatou pontuações } \\
\text { no PHQ-9 indicativas de transtorno } \\
\text { depressivo maior (TDM). }\end{array}$ & $\begin{array}{l}\text { Quanto mais grave a } \\
\text { demência (Alzheimer) pior a } \\
\text { Qualidade de Vida dos } \\
\text { cuidadores e pacientes, } \\
\text { sugerindo que a detecção e o } \\
\text { tratamento precoce do } \\
\text { Alzheimer podem reduzir o } \\
\text { fardo da doença para os } \\
\text { cuidadores, pacientes e } \\
\text { sociedade em geral. }\end{array}$ & V \\
\hline 9 & Du et al. & $\begin{array}{l}\text { Descrever a Qualidade de Vida } \\
\text { relacionada à saúde (QVRS) e } \\
\text { identificar seus preditores para } \\
\text { cuidadores familiares chineses de } \\
\text { idosos com deficiência. } \\
\text { Método: quantitativo. }\end{array}$ & $\begin{array}{l}\text { A Sobrecarga subjetiva do cuidador foi } \\
\text { negativamente relacionada a todas as } \\
\text { subescalas da QVRS do cuidador e, } \\
\text { portanto, foi o mais forte preditor de QVRS. }\end{array}$ & $\begin{array}{l}\text { O apoio social adicional é } \\
\text { importante na diminuição do } \\
\text { impacto do cuidado na } \\
\text { QVRS. }\end{array}$ & V \\
\hline 10 & Lino et al. & $\begin{array}{l}\text { Verificar, entre cuidadores } \\
\text { familiares, a prevalência de } \\
\text { Sobrecarga e os fatores } \\
\text { associados a ela em uma região } \\
\text { pobre e violenta do Rio de } \\
\text { Janeiro, Brasil. } \\
\text { Método: transversal, } \\
\text { quantitativo. }\end{array}$ & $\begin{array}{l}\text { As seguintes características dos idosos se } \\
\text { associaram à Sobrecarga: idade }(\mathrm{OR}=0,94 \text {; } \\
\mathrm{p}<0,002) \text {, depressão } \\
(\mathrm{OR}=2,59 ; \mathrm{p}<0,005) \text { e declínio cognitivo } \\
(\mathrm{OR}=3,19 ; \mathrm{p}<0,03) \text {. Em relação aos } \\
\text { fatores dos cuidadores familiares, apenas } \\
\text { apoio social manteve a relevância } \\
(\mathrm{OR}=2,35 ; \mathrm{p}<0,005) \text {. }\end{array}$ & $\begin{array}{l}\text { Investigar e tratar depressão e } \\
\text { demência em idosos, assim } \\
\text { como prover apoio aos seus } \\
\text { cuidadores podem contribuir } \\
\text { para o manejo efetivo da } \\
\text { Sobrecarga de cuidadores } \\
\text { familiares, melhorando a } \\
\text { qualidade do cuidado e a } \\
\text { saúde de ambos. }\end{array}$ & V \\
\hline 11 & Anjos et al. & $\begin{array}{l}\text { Averiguar a associação entre o } \\
\text { apoio social e a Qualidade de } \\
\text { Vida de cuidadores familiares de } \\
\text { idosos dependentes no domicilio. } \\
\text { Método: transversal, descritivo, } \\
\text { quantitativo. }\end{array}$ & $\begin{array}{l}\text { A maioria dos cuidadores foi de mulheres, } \\
\text { cuidava em tempo integral e apresentava } \\
\text { Sobrecarga moderada a severa. A maioria } \\
\text { dos cuidadores está satisfeita com as } \\
\text { relações sociais e o apoio social recebido. } \\
\text { Verifica-se que a Sobrecarga e o tempo de } \\
\text { cuidado correlacionaram com o domínio } \\
\text { relações sociais, que associa-se ao apoio } \\
\text { social e, consequentemente, à redução da } \\
\text { Qualidade de Vida. }\end{array}$ & $\begin{array}{l}\text { O apoio social aos cuidadores } \\
\text { é importante para prevenir } \\
\text { implicações à saúde, } \\
\text { Sobrecarga, desgaste } \\
\text { biopsicossocial e } \\
\text { proporcionar condições } \\
\text { favoráveis à Qualidade de } \\
\text { Vida desses, ao permitir } \\
\text { maior liberdade para } \\
\text { desenvolver suas atividades } \\
\text { cotidianas. }\end{array}$ & V \\
\hline 12 & Coura et al. & $\begin{array}{l}\text { Avaliar a Qualidade de vida dos } \\
\text { cuidadores de octogenários e } \\
\text { identificar os domínios } \\
\text { correlacionados com a Qualidade } \\
\text { de Vida e saúde. } \\
\text { Método: transversal, } \\
\text { quantitativo. }\end{array}$ & $\begin{array}{l}\text { As pontuações dos domínios foram: } \\
\text { ambientais }(59,43) \text {, psicológico }(59,01) \text {, } \\
\text { relações sociais }(46,77) \text { e físico }(43,86), \\
\text { cujos valores indicam insatisfação dos } \\
\text { sujeitos no que se refere aos aspectos } \\
\text { relacionados à Qualidade de Vida. O } \\
\text { domínio ambiental foi correlacionado com } \\
\text { Qualidade de Vida e o domínio físico com } \\
\text { percepções de saúde; a segurança diária e os } \\
\text { aspectos da dor tiveram as maiores } \\
\text { correlações com a qualidade de vida. }\end{array}$ & $\begin{array}{c}\text { A percepção dos cuidadores } \\
\text { quanto à Qualidade de Vida } \\
\text { não era boa. Os domínios } \\
\text { ambiental e físico tiveram as } \\
\text { maiores correlações com } \\
\text { Qualidade de Vida e saúde. }\end{array}$ & V \\
\hline
\end{tabular}

Fonte: Autores (2021). 


\section{Discussão}

\section{Cuidador Familiar de Idoso}

Nos últimos anos, a população mundial está passando por um processo de envelhecimento populacional, isso gera consequências de curto a longo prazo. O envelhecimento afeta a vida do ser humano de diferentes formas, ocasionando limitações funcionais, que em alguns casos torna-se necessário o auxílio de um cuidador, normalmente tal responsabilidade recai sobre algum familiar do idoso. O cuidador familiar exercer um papel essencial na vida do idoso, contudo, ainda são poucos os estudos voltados para esse grupo, ainda mais que tratem da Qualidade de Vida e Sobrecarga do cuidador, que de certa maneira, acabam ficando vulneráveis a diversas doenças, tanto físicas como mentais. Percebemos que, quanto ao ano de publicação, os resultados evidenciam um aumento de publicações entre os anos de 2018 e 2019, com queda em 2020 e 2021 , podendo ser devido a pandemia de covid-19, que impossibilitou a produção de mais trabalhos, já que no ano de 2019 foram encontrados um número maior de publicações. Em relação ao local, é importante destacar que mais da metade dos estudos foram realizados no Brasil, assim, os dados demonstram de maneira clara a realidade brasileira relacionada ao cuidador, como está sendo a Qualidade de Vida e Sobrecarga dessa população no país, bem como, as melhorias no ambiento da saúde e nos aspectos sociais que precisam ser implantadas para que essa realidade mude em longo prazo, já que a tendência é que a população idosa aumente, necessitando de mais apoio nesse processo de cuidado. Além disso, o método mais utilizado foi de estudo transversal, uma ferramenta de grande utilidade para a descrição de características da população e para a identificação de grupos de risco, assim como, a compreender como esta modificação de saúde abrange os sinais e sintomas relacionados (Sitta et al., 2010).

Os dados encontrados nos resultados permitiram analisar o perfil do principal cuidador responsável pelo idoso, primeiramente, as maiorias dos estudos apontaram que a mulher é a principal responsável pelo cuidado, recaindo sobre ela a incumbência de cuidar do idoso. Assumir os cuidados, para as mulheres, na maioria das vezes, é visto como algo natural e que se constrói como mais um papel doméstico culturalmente e socialmente aceito, que passa entre as gerações do núcleo familiar (ARAÚJO et al., 2019).

Somente no estudo de Montgomery et al. (2018) realizado com cuidadores familiares de idosos com a Alzheimer, a quantidade de homens responsáveis pelo cuidado foi maior, a justificativa para tal, foi o fato da pesquisa ter sido feita de forma online e os mesmos apresentarem vantagens socioeconômicas, como o fato da maioria estar empregado, refletindo em uma maior motivação, capacidade física e acesso à Internet. Considerando que a representação relativamente grande de cuidadores do sexo masculino contrasta com outros estudos internacionais semelhantes de cuidadores de idosos com demência, em que cuidadoras do sexo feminino eram a maioria. Já relacionado a idade, quando se agrupa as faixas etárias mais frequentes, que seria entre 50 a 69 anos, evidencia-se a maior representatividade de adultos mais velhos e de idosos. A idade dos cuidadores é uma variável relevante, uma vez que, frequentemente, o cuidador pertence à mesma geração dos indivíduos cuidados ou estão entrando nessa fase da vida, o que pode repercutir em sérios problemas de saúde a longo prazo para o cuidador (Anjos et al., 2015). E os idosos que cuidam informalmente de outros idosos estão sujeitos a dois tipos de riscos: (1) riscos decorrentes do exercício da função de cuidador e (2) riscos decorrentes do próprio envelhecimento, o que pode acarretar consequências para a saúde de ambos, tanto para o idoso cuidado, como para o responsável pelo cuidado (Flesch et al., 2020). A maioria dos cuidadores familiares possuía baixa escolaridade, além de que alguns estudos não descreveram essa informação, que é algo muito importante relacionado ao cuidado, como afirmar Lino et al. (2016), o baixo letramento em saúde pode ter um impacto direto sobre o cuidado, em decorrência das dificuldades na compreensão do processo de adoecimento do idoso e da falta de acesso a informações, podendo gerar Sobrecarga. Dos estudos que descreveram o estado civil do cuidador, a maioria dos entrevistados era casado ou vivia em união estável, outro fator que pode gerar repercussão na vida do cuidador, relacionado ao seu autocuidado, pois quando o cuidador é do sexo feminino, acumulam outros papéis, tais como: o de mãe, esposa, cuidadora 
de pessoas dependentes e alguns casos ainda precisam trabalhar fora de casa (Couto; Caldas \& Castro, 2019). Sobre a relação do cuidador com o idoso, a maioria dos participantes das pesquisas analisadas eram filhos que ficaram com a incumbência de cuidar dos pais na velhice. Como ressaltam Haya et al. (2019) que realizaram sua pesquisa no Japão, semelhante ao que acontece com outras culturas asiáticas, o papel de cuidar repousa principalmente sobre as mulheres na família, seja o cônjuge ou filho adulto. Ressalta-se que além dos filhos, outro responsável muito frequente pelo cuidado foram os cônjuges, mesmo aqueles que tem idade próxima do idoso cuidado, já que é comum o papel ser assumido pelo companheiro, por ser a pessoa mais próxima do idoso, fator que faz com que o restante da família aceite facilmente essa escolha, além de estar socialmente estabelecido que entre o casal deve haver cuidado mútuo (Flesch et al., 2020). Outro fato relevante, é que a maioria dos idosos que necessitavam de cuidados estava na faixa etária acima de 80 anos, estando relacionado ao aumento da expectativa de vida da população, bem como das comorbidades que geram uma maior fragilidade do idoso. Nestes casos, quando ocorre déficit físico, a incapacidade funcional pode prejudicar a independência e autonomia dessas pessoas, condição que compromete, além da saúde, a Qualidade de Vida e, não raro, demanda cuidados permanentes (Coura et al., 2015). Além disso, os idosos octogenários acabam por demonstrar mais sinais de senescência e senilidade relacionadas ao envelhecimento, pois outro fator observado nos estudos que descreveram as doenças presentes na população que necessitava de cuidados, a demência foi a mais citada. A demência do Alzheimer (DA) é uma doença neurodegenerativa progressiva que representa um fardo significativo para os pacientes, cuidadores, sistemas de saúde e a sociedade (Montgomery et al., 2018). Os idosos com esse diagnóstico acabam por precisar de mais cuidados, à medida que a doença progride, necessitando assim uma maior assistência nas atividades de vida diária.

\section{Sobrecarga do Cuidador Familiar de Idoso}

Quanto a análise da Sobrecarga e Qualidade de vida, 3 (25\%) investigaram apenas a Sobrecarga $(3,5,10) ; 2$ (16,7\%) pesquisaram apenas a Qualidade de vida (11,12), os demais analisaram ambas as informações. (Quadro 1). Acerca da Sobrecarga, 10 estudos avaliaram como os cuidadores se sentiam, em 6 estudos (60\%) Sobrecarga moderada (2,3,4,8,9,10), 2 (20\%) Sobrecarga alta $(1,5)$ e $2(20 \%)$ sobrecarga baixa $(6,7)$. Além disso, dentre as dificuldades relatadas pelos cuidadores que afetavam a vida, $7(58,4 \%)$ relataram falta de apoio e tempo para si $(3,4,6,7,9,10,12), 6(50 \%)$ apresentavam alguma comorbidade $(1,3,4,5,8,9), 4(33,3 \%)$ Sobrecarga financeira $(4,7,11,12)$. (Quadro 1)

A maioria dos estudos apontou que os cuidadores relataram uma Sobrecarga moderada e a minoria relatou Sobrecarga alta. Kehoe et al. (2019), Lino et al. (2016) justificaram esses valores como dados mascarados, uma vez que os cuidadores repassaram informações incorretas, e que os mesmos, queriam camuflar a realidade diante das pessoas de fora. E como o cuidado era realizado na maioria das vezes por mulheres, não queriam demonstrar fraqueza ou ingratidão relacionado ao idoso cuidado.

Como ressaltam Araújo et al. (2019), as entrevistadas ao se depararem com opções que evidenciavam mais Sobrecarga, interpretavam isso como lamentação e protesto e não queriam passar essa impressão, porquanto se sentiam culpadas. Todavia, o pouco tempo reservado para o lazer, as relações pessoais e o autocuidado desmentiam as alegações.

Mesmo que a Sobrecarga seja moderada, ainda é algo que precisa ser trabalho, pois se acredita que isso se deve, principalmente, à Sobrecarga de trabalho devido o ato de cuidar, somado as atribuições domésticas, como cuidar dos filhos, uma vez que, como fora reiteradamente colocado, a maioria são mulheres, historicamente responsáveis por estas atividades (Coura et al., 2015).

Como demonstra Kehoe et al. (2019), os resultados evidenciaram que na análise multivariada com 541 cuidadores, aqueles que cuidam de idosos que precisam de mais ajuda nas atividades instrumentais da vida diária têm maior probabilidade de vivenciar alta Sobrecarga do cuidado. 
Além disso, a Sobrecarga do cuidador afeta diretamente a Qualidade de Vida do idoso cuidado, pois, estudos de Tessitore et al. (2018) com idosos que apresentavam doença de Parkinson e seus cuidadores, observaram que a Qualidade de Vida do paciente está frequentemente associada à Sobrecarga do cuidador, o que deve ser levado em consideração no manejo a longo prazo do idoso.

E também foi possível associar a relação direta entre a Sobrecarga e a Qualidade de Vida. Nos estudos de Anjos et al. (2015), identificou-se que, quanto maior a Sobrecarga do cuidador e tempo (meses) dedicado ao cuidar do idoso, pior a percepção da qualidade vida em todos os domínios do WHOQOL-bref e, especialmente, no domínio meio ambiente, o que pode estar associado com o déficit de apoio informal e inexistência do apoio formal recebido.

Ademais, na análise dos resultados dos artigos foi possível elencar as dificuldades enfrentadas pelos cuidadores familiares. A mais observada foi a falta de apoio e tempo para si, os cuidadores eram os principais responsáveis pelo cuidado, o que por sua vez ocasionava a Sobrecarga, afetando a Qualidade de Vida, pois devido ao papel exercido, eles não possuíam tempo para realizarem o autocuidado.

Nos estudos de Du et al. (2017), os cuidadores que recebiam suporte social demonstraram uma melhor Qualidade de Vida, uma possível razão para isso pode ser que as pessoas que desfrutam de maior apoio subjetivo tendem a usar estratégias de enfrentamento mais eficazes, em comparação com aquelas que recebem menos apoio. Os resultados de Lino et al. (2016) demonstram uma relação entre as diferentes dimensões de apoio social, assim, observou-se um maior impacto da interação social positiva e do apoio emocional na Sobrecarga de cuidadores, verificou que o baixo nível de apoio social acarreta Sobrecarga aos cuidadores. Outro fator avaliado como o preditor de impasse para os cuidadores foi a presença de comorbidades, que podem ser anteriores ao papel de cuidador, ou surgirem após o início do cuidado. Visto que os cuidadores costumam ser mais velhos e suas próprias comorbidades estão associadas a problemas de saúde emocional e a Qualidade de Vida (Kehoe et al., 2019).

Ser responsável pelo cuidado gera Sobrecarga financeira, em razão dos gastos comuns ou devido ao fato de muitos precisarem deixar de exercer atividades remuneradas para se responsabilizar pelo cuidado do idoso, passando a depender exclusivamente da aposentadoria, que nem sempre consegue cobrir os gastos realizados.

Haya et al. (2019), encontrou nos seus dados qualitativos evidências que apoiaram este achado e mostraram que o cuidado afetou a condição física, as emoções, a vida social e as finanças dos cuidadores.

Assim, os cuidadores de idosos são chamadas "vítimas ocultas", vez que em várias situações, além de conviver com o isolamento, solidão, Sobrecarga de atividades, responsabilidades de cuidar e dificuldades financeiras, ainda lidam com o seu processo de envelhecimento (Anjos et al., 2015).

\section{Qualidade de Vida do Cuidador Familiar de Idoso}

A respeito da análise da Qualidade de vida e Sobrecarga dos cuidadores, mesmo que alguns estudos não tenham pesquisado ambas as informações, os resultados encontrados foram significativos, pois a grande maioria dos autores relacionaram os dois fatores, descrevendo que a Sobrecarga afeta diretamente a Qualidade de Vida do cuidador.

Em relação à Qualidade de Vida dos cuidadores, dos 9 estudos que analisaram esse constructo, em 5 (55\%) os informantes a consideram insatisfatória $(1,4,9,11,12)$, em 3 (33\%) mediana $(2,6,8), 1$ (11\%) consideraram a Qualidade de Vida boa (7). (Quadro 1) Assim, sobre a Qualidade de Vida do cuidador, a maioria dos entrevistados a consideravam insatisfatória/diminuída. O estudo de Du et al. (2017) com 766 cuidadores chineses de Pequim apresentou, nos resultados encontrados, que muitos cuidadores familiares de idosos experimentavam uma Qualidade de Vida relacionada a saúde diminuída. Como cuidadores primários, os membros da família acabam arcando com o dever de cuidar dos idosos em casa, que, dependendo das condições de saúde do idoso, pode ser uma tarefa cansativa, e esses cuidadores familiares têm muitos 
riscos à saúde e menor Qualidade de Vida em comparação com outros grupos de não cuidadores (Haya et al., 2019).

Isso pode ser devido ao papel exercido pelo cuidador, que possui uma tarefa árdua, caracterizada por ser repetitiva, incessante e, geralmente, acumulada com outras atividades domésticas e profissionais; resultando em esgotamento, isolamento social, afetando o autocuidado e como consequência a Qualidade de Vida (Coura et al., 2015).

\section{O Papel da Enfermagem}

Como observado, várias são as formas/estratégias de cuidado utilizadas pelo cuidador familiar de idoso, pois o seu trabalho vai além da vontade de querer cuidar do seu familiar, dada a complexidade que o cuidado exige. Envolve conhecimento, desenvolvimento de habilidades, iniciativas para a promoção, tratamento e recuperação da saúde do idoso; trabalho este definido pelos próprios cuidadores como "difícil", que exige paciência, amor e até mesmo renúncia de seu projeto de vida. Tudo isso têm contribuído para que os cuidadores vivenciem diariamente Sobrecarga física, emocional e social no cotidiano de cuidado.

Assim, as famílias precisam de apoio de profissionais, como os da Enfermagem, diante dessas situações, para que possam organizar e estabelecer uma rotina de cuidados que envolva o maior número possível de membros da família ou mesmo de amigos e vizinhos.

Por conseguinte, os cuidadores familiares necessitam de recursos assistenciais do sistema de saúde, suporte emocional e aprendizagem de processos. São necessárias pesquisas sobre planos de cuidados interprofissionais aos cuidadores familiares no âmbito de políticas e serviços de atenção domiciliar (Couto; Caldas \& Catro, 2019).

Logo, a Enfermagem apresenta um poder de contribuição considerável, pois através da implementação de medidas de promoção da saúde pode, efetivamente, melhorar a vida desses indivíduos. (Araújo et al, 2019).

Uma das medidas de promoção a saúde é a Cartilha, que é uma tecnologia educacional cuidativa, sendo esta eleita como produto deste estudo, com linguagem acessível, principalmente para os cuidadores de baixa escolaridade, com intuito de contribuir para o incremento dos conhecimentos por parte do público alvo.

\section{Considerações Finais}

A expectativa de vida da população mundial aumentou de maneira vertiginosa, algo que antes era um fenômeno, passou a ser parte da realidade de diversos países, que precisaram se adequar à crescente demanda de cuidados que essa parcela da população precisará, à medida que alcance idades mais avançadas.

Com isso, cresceu a demanda por cuidados e um maior acompanhamento nas atividades de vida diária do idoso, esse papel quase sempre é realizado pela família, segundo os dados colhidos, a responsabilidade na maioria das vezes recai sobre a mulher, que passa a exercer diversas funções no contexto familiar.

Dessa maneira, os resultados revelaram que exercer o papel de cuidador afeta diretamente a Qualidade de Vida dos responsáveis pelo cuidado de forma negativa, gerado principalmente pela Sobrecarga, já que normalmente as atividades acabam por ser exercidas pelo cuidador principal. Essa Sobrecarga ocasiona um maior adoecimento para o cuidador, afastamento social e dificuldades econômicas.

Para que seja possível uma mudança nessa realidade, devem ser realizas medidas visando a melhoria das condições de vida do cuidador responsável pelo idoso, como ambientes de apoio, por exemplo, centro-dia para os idosos, atendimento e acompanhamento individualizado para os cuidadores, capacitação para os cuidadores e políticas públicas efetivas voltadas para o idoso.

Havendo a necessidade de ações mais efetivas voltadas para os cuidadores familiares, evidenciando a necessidade do acompanhamento multiprofissional. As evidências produzidas pela RI mostraram a relevância da atuação do enfermeiro na 
orientação, acompanhamento e suporte ao cuidador, visto que avalia as necessidades do idoso e da família, levando em consideração as diferentes realidades vivenciadas pelos cuidadores familiares de idosos dependentes em domicílio. Tendo em vista a sua responsabilidade em especial na atenção ao cuidador que recebe a incumbência de cuidar e nem sempre recebe suporte externo como a capacitação para a realização das tarefas, cabe a enfermagem realizar o acompanhamento tanto dos idosos, como dos seus cuidadores, principalmente na atenção primária em saúde, e realizar ações que visem a promoção da Qualidade de Vida de ambos, bem como capacitar esses cuidadores familiares, para que seja possível a redução da Sobrecarga.

Portanto, o presente estudo conseguiu alcançar os objetivos propostos, e busca assim auxiliar nas discussões sobre esse grupo populacional, os cuidadores familiares, que acabam sendo extremante vulneráveis, devido a dupla, tripla jornada exercida além do cuidado, precisando que mais pesquisas futuras sejam realizadas para avaliar quais medidas são mais efetivas para a melhoria da Qualidade de Vida, bem como, a diminuição da Sobrecarga. As limitações para melhor execução do presente estudo se deram pelo número reduzido de publicações concernentes ao tema, em especial no período de 2020 e 2021 provavelmente devido a pandemia do Coronavírus, além de alguns estudos não estarem disponíveis na íntegra.

Sugere-se que pesquisas futuras sejam conduzidas com uma abordagem mais geral, independentemente da idade do idoso, uma vez que a necessidade de cuidadores é indispensável para idosos, e o nível desse compromisso é que interfere na sobrecarga do cuidador e, consequentemente, na sua qualidade de vida.

O produto proposto por este estudo é uma Cartilha de Orientações de Enfermagem para o Cuidador de Idosos da Quarta Idade, com itens selecionados após a obtenção e análise das discussões encontradas nesta revisão, a partir das demandas apresentadas pelos cuidadores, acerca da Sobrecarga, resultante do impacto do cuidado no seu dia-a-dia.

\section{Referências}

Anjos, K. F, Boery, R. N. S. O., Santos, V. C., Boery, E. N., Silva, J, K \& Rosa, D. O. S. (2018). Fatores Associados à Qualidade de Vida de Cuidadores Familiares de Idosos. Ciencia y enfermería, 24, 17.https://dx.doi.org/10.4067/s0717-95532018000100217

Araújo, M. G. O et al. (2019) Caring for the carer: quality of life and burden of female caregivers. Revista Brasileira de Enfermagem, 72, 728-736. https://doi.org/10.1590/0034-7167-2018-0334

Brasil. Ministério da Saúde. (2008) Guia prático do cuidador. bvsms.saude.gov.br/bvs/publicações/guia_pratico_cuidador.pdf.

Brasil. Ministério da Saúde. (2018) Estudos apontam que 75\% dos idosos usam apenas o SUS. Brasil., http:// portalms.saude.gov.br/ notícias/agencia-saude/ 44451-estudo-aponta-que-75-dos-idosos-usam-apenas-o-sus.

Coura, A. S., Alves, C. N., Alves, F. P, da Silva, J. A., de França, I. S. X, \& Silva, K. K. A. M (2015). Qualidade de vida de cuidadores de octogenários: um estudo com o WHOQOL-BREF. Investigación y Educación en Enfermería, 33 (3), 529-538. https://doi.org/10.17533/udea.iee.v33n3a17

Couto, A. M., Caldas, C. P \& Castro, E. A. B. (2019). Home care for dependent elderly patients by caregivers with overload and stress / Cuidado domiciliar a idosos dependentes de cuidadores familiares com sobrecarga e desconforto emocional. Revista de Pesquisa Cuidado é Fundamental Online, 11(4), 944-950. https://doi.org/10.9789/2175-5361.2019.v11i4.944-950

Crossetti, M. G. O. (2012). Revisión integrativa de la investigación en enfermería, el rigor científico que se le exige. Revista Gaúcha de Enfermagem, 33, 1011. https://doi.org/10.1590/S1983-14472012000200002.

Dias, L. B., Castiglioni, L., Tognola, W. A., \& Bianchin, M. A. (2018). Sobrecarga no cuidado de paciente idoso com demência. Revista Kairós — Gerontologia, 21(1), 169-190. http://dx.doi.org/10.23925/2176-901X.2018v21i1p169-190

Du, J., Shao, S., Jin, G. H., Qian, C. G., Xu, W., \& Lu, X. Q. (2017). Factors associated with health-related quality of life among family caregivers of disabled older adults: a cross-sectional study from Beijing. Medicine, 96(44), e8489. https://doi.org/10.1097/MD.0000000000008489

Flesch, L. D et al.(2020) Elderly Who Care for Elderly: DoubleVulnerability and Quality of Life. Paidéia. 30. https://doi.org/10.1590/1982-4327e3003.

Gomes, W. D \& Resck, Z. M. R.(2009). A percepção dos cuidadores domiciliares no cuidado a clientes com sequelas neurológicas. Rev. enferm. UERJ. 17(4): 496-501.

Grupo Anima Educação. (2014). Manual de Revisão Bibliográfica Sistemática Integrativa: a pesquisa baseada em evidências.

Haya, M., Ichikawa, S., Wakabayashi, H. \& Takemura, Y. (2019). Family Caregivers' Perspectives for the Effect of Social Support on their Care Burden and Quality of Life: A Mixed-Method Study in Rural and Sub-Urban Central Japan. The Tohoku journal of experimental medicine, $247(3)$, 197-207. https://doi.org/10.1620/tjem.247.197 
Research, Society and Development, v. 10, n. 10, e478101018931, 2021

(CC BY 4.0) | ISSN 2525-3409 | DOI: http://dx.doi.org/10.33448/rsd-v10i10.18931

Hoffmann, T. \& Worrall, L. (2004). Designing effective written health education materials: considerations for health professionals. Disability and rehabilitation, 26(19), 1166-1173. https://doi.org/10.1080/09638280410001724816

Instituto Brasileiro de Geografia e Estatística. (2020) Em 2019, expectativa de vida era de 76,6 anos. https:// agenciadenoticias.ibge.gov.br/agencia-sala-deimprensa/2013-agencia-de-noticias /releases/29502-em-2019-expectativa-de-vida-era-de-76-6-anos.

Kehoe, L. A., Xu, H., Duberstein, P., Loh, K. P., Culakova, E., Canin, B., Hurria, A., Dale, W., Wells, M., Gilmore, N., Kleckner, A. S., Lund, J., Kamen, C., Flannery, M., Hoerger, M., Hopkins, J. O., Liu, J. J., Geer, J., Epstein, R., \& Mohile, S. G. (2019). Quality of Life of Care givers of Older Patients with Advanced Cancer. Journal of the American Geriatrics Society, 67(5), 969-977. https://doi.org/10.1111/jgs.15862

Lino, V. T. S., Rodrigues, N. C. P., Camacho, L. A. B., O’Dwyer, G., Lima, I. S., Andrade, A. K. N \& Atie, S. (2016). Prevalência de sobrecarga e respectivos fatores associados em cuidadores de idosos dependentes, em uma região pobre do Rio de Janeiro, Brasil. Cad. Saúde Pública, 32https://doi.org/10.1590/0102$311 \times 00060115$

Montgomery, W., Goren, A., Kahle-Wrobleski, K., Nakamura, T., \& Ueda, K. (2018). Alzheimer's disease severity and its association with patient and caregiver quality of life in Japan: results of a community-based survey. BMC geriatrics, 18(1), 141. https://doi.org/10.1186/s12877-018-0831-2

Organização Mundial da Saúde. Relatório mundial de envelhecimento e saúde. OMS, 2015.

Salbego, C., et al. (2018). Care-educational technologies: an emerging concept of the praxis of nurses in a hospital context. Revista Brasileira de Enfermagem, 71, 2666-2674. https://doi.org/10.1590/0034-7167-2017-0753.

Sitta, E. I., Arakawua-Belaunde, A. M., Caldana, M. L. \& Salles-Peres, S. H. C. (2010). A contribuição de estudos transversais na área da linguagem com enfoque em afasia. Revista CEFAC, 12 (6). https://doi.org/10.1590/S1516-18462010005000086

Souza, M. T., Silva, M. D. \& Carvalho, R. (2010). Revisão integrativa: o que é e como fazer. Einstein, 8(1), 102-106. https://doi.org/10.1590/s1679$45082010 \mathrm{rw} 1134$

Tessitore, A., Marano, P., Modugno, N., Pontieri, F. E., Tambasco, N., Canesi, M., Latorre, A., Lopiano, L., Sensi, M., Quatrale, R., Solla, P., Defazio, G., Melzi, G., Costanzo, A. M., Gualberti, G., di Luzio Paparatti, U., \& Antonini, A. (2018). Caregiver burden and its related factors in advanced Parkinson's disease: data from the PREDICT study. Journal of neurology, 265(5), 1124-1137. https://doi.org/10.1007/s00415-018-8816-9 